\title{
Mutual eye gaze facilitates person categorization for typically developing children, but not for children with autism
}

\author{
Elizabeth Pellicano \\ Institute of Education, London, England \\ and University of Western Australia, Crawley, Western Australia, Australia \\ AND \\ C. NeIL Macrae \\ University of Aberdeen, Aberdeen, Scotland
}

\begin{abstract}
Previous investigations of gaze processing in autism have demonstrated a pattern of intact and impaired performance. Although individuals with autism are capable of discriminating another's gaze, they fail to interpret gaze direction, especially within the context of sociocommunicative (i.e., mentalistic) interactions. Extending this general line of inquiry, we explored whether typical children and children with autism spectrum disorder (ASD) were influenced by gaze direction in a task that demanded a core person-related judgment-namely, sex categorization. The results revealed that typically developing school-aged children were faster to classify faces by sex when targets displayed direct rather than averted gaze, or when the eyes were closed. This was not the case, however, for children with ASD, whose responses were unaffected by gaze direction. These findings suggest that difficulties in gaze processing in autism extend beyond sociocommunicative inferences to include basic person-perception judgments.
\end{abstract}

Detecting and interpreting another's direction of gaze is pivotal to interpersonal interactions. Mutual eye gaze, in particular, serves to signal the likely flavor of a social interaction, be it nasty or nice, to initiate and regulate social communication, and to provide important information about the mental states of others (Nummenmaa $\&$ Calder, 2009). It is perhaps of no surprise, then, that adults are exquisitely sensitive to gaze direction (von Grünau \& Anston, 1995), that such sensitivity emerges early in ontogenesis (Farroni, Mansfield, Lai, \& Johnson, 2003), and that atypicalities in gaze processing are a defining feature of autism (American Psychiatric Association [APA], 2000), a developmental condition characterized by profound difficulties in social reciprocity and communication.

In typical development, infants appear to be born with a preparedness to respond to gaze cues. Newborns prefer looking at faces showing direct gazes over those showing averted gazes (Farroni, Csibra, Simion, \& Johnson, 2002) or those with closed eyes (Bakti, Baron-Cohen, Wheelwright, Connellan, \& Ahluwalia, 2000). Threemonth-olds smile less when an adult looks away and resume smiling when the adult reinitiates eye contact (Hains \& Muir, 1996), and 4-month-olds rapidly shift their own gaze toward a target only when such shifts are preceded by a period of mutual eye gaze (Farroni et al., 2003). Re- cent work has revealed that this early sensitivity to eyegaze direction, particularly mutual gaze, holds special significance during infant-adult interactions. Senju and Csibra (2008) examined the gaze following of 6-monthold infants as they observed a person looking toward a toy located on either the left or the right. Infants followed the adult's eye movement only when it was preceded by direct eye contact, suggesting an early awareness of the ostensive nature of gaze cues. In another study, 9-month-olds observed a face either always looking toward an object or always looking away from it. Infants preferred to look at an adult making object-directed shifts in eye gaze but, again, only when such shifts were preceded by direct gaze (Senju, Csibra, \& Johnson, 2008). Both findings indicate an apparent "eye-contact effect" in which perceived eye contact influences subsequent processing of another's sociocommunicative intentions.

Direct eye gaze clearly affords advantages for children's developing mentalizing skills (e.g., Baron-Cohen, 1995). Yet research with adults and children suggests that mutual gaze also affords additional benefits. Notably, it shapes more basic aspects of the person-perception process. Macrae, Hood, Milne, Rowe, and Mason (2002) demonstrated that gaze direction moderates the efficiency of person construal. Specifically, adults are faster to judge the gender of a face when the target's eyes are directed straight ahead

E.Pellicano, 1.pellicano@ioe.ac.uk 
than when they are averted or closed. Also, they are more accurate in recognizing previously seen faces that display direct gaze (Mason, Hood, \& Macrae, 2004), a memorial effect that also has been reported in 4-month-olds (Farroni, Massaccesi, Menon, \& Johnson, 2007) and children (Hood, Macrae, Cole-Davies, \& Dias, 2003). Mutual gaze, therefore, not only conveys important mentalistic information about others, but also offers an additional advantage: It facilitates the extraction of core person-relevant knowledge (e.g., sex, identity) from faces.

In autism, deviant patterns of reciprocal eye gaze are a striking feature (APA, 2000; see Nation \& Penny, 2008) that may be one of its earliest (detectable) manifestations (e.g., Baron-Cohen et al., 1996). Children with autism engage less in direct eye-to-eye contact (Sigman, Mundy, Sherman, \& Ungerer, 1986) and fixate less on the eyes during spontaneous viewing of faces than do typical individuals (e.g., Pelphrey et al., 2002; but see Fletcher-Watson, Leekam, Benson, Frank, \& Findlay, 2009). Furthermore, they fail to monitor the target of another person's gaze during joint visual attention (e.g., Leekam, Hunnisett, \& Moore, 1998). In gaze-reading tasks, children with autism do not interpret gaze direction as a cue to another's mental state (Baron-Cohen, Campbell, Karmiloff-Smith, Grant, \& Walker, 1995), and autistic adults show difficulties inferring another's mental state from viewing expressions in the eyes (Baron-Cohen, Jolliffe, Mortimore, \& Robertson, 1997). This failure to understand the mentalistic significance of the eyes is thought to be instrumental in causing autistic children's theory-of-mind difficulties (Baron-Cohen, 1995).

Research has shown, however, that individuals with autism are not completely insensitive to gaze cues. Older and more able children with autism do demonstrate basic knowledge about eyes and seeing (Tan \& Harris, 1991). They also are able to discriminate accurately where another person is looking (Leekam, Baron-Cohen, Perrett, Milders, \& Brown, 1997; Wallace, Coleman, Pascalis, \& Bailey, 2006) and can automatically shift their visual attention in response to gaze cues in a manner similar to that of typical individuals (see Nation \& Penny, 2008, for a review).

Overall, these findings suggest that individuals with autism show no specific impairment in the ability to detect and orient automatically to another's gaze direction. Rather, the primary difficulty appears to lie in the ability to extract information from the eyes for specific sociocommunicative purposes (Baron-Cohen, 1995; Nation \& Penny, 2008). This might suggest that the difficulty with eye gaze in autism derives from the sociocommunicative functions to which eye gaze relates - functions thought by some to be the core impairment in autism. No study, however, has yet considered whether gaze-processing atypicalities in autism also extend to other potential eye-gaze advantages among the typical population, such as those seen in the more basic features of the person-perception process (Macrae \& Bodenhausen, 2000).

In this inquiry, we investigated, therefore, the impact that gaze cues exert on the efficiency of person categorization in both typical development and autism. We anticipated that typical school-age children would display a response time (RT) advantage when faces display direct gaze during a sex-categorization task, as is the case for adults (Macrae et al., 2002). We also hypothesized that, if one considers gaze atypicalities in autism to be attributable to specific sociocommunicative difficulties (e.g., Baron-Cohen, 1995), such atypicalities should be restricted to mentalistic contexts, and basic person-perception processes should remain unaffected. Accordingly, like their typical counterparts, children with autism should demonstrate facilitated categorical performance when targets display direct gaze. If, however, children with autism have more basic difficulties processing gaze cues, such an effect should fail to emerge.

\section{METHOD}

\section{Participants and Design}

Descriptive information is provided in Table 1. Twenty-four children with an autism spectrum disorder (ASD; 23 boys; $M=$ 10 years, 2 months; $S D=1$ year, 9 months) were recruited in Perth,

Table 1

Descriptive Statistics for Chronological Age, Nonverbal IQ, Verbal IQ, Social Communication Questionnaire (SCQ) Scores (Rutter, Bailey, \& Lord, 2003), and Subdomain Scores on the Autism Diagnostic Interview-Revised (ADI-R; Lord, Rutter, \& Le Couteur, 1994)

\begin{tabular}{|c|c|c|c|c|c|c|}
\hline \multirow[b]{3}{*}{ Measure } & \multicolumn{6}{|c|}{ Group } \\
\hline & \multicolumn{3}{|c|}{$\operatorname{ASD}(n=24)$} & \multicolumn{3}{|c|}{ Typical $(n=24)$} \\
\hline & $M$ & $S D$ & Range & $M$ & $S D$ & Range \\
\hline Chronological age (months) & 122.29 & 21.79 & $96-163$ & 120.25 & 20.58 & $96-168$ \\
\hline Nonverbal IQ & 100.00 & 14.29 & $74-131$ & 97.29 & 11.28 & $78-131$ \\
\hline Verbal IQb & 96.12 & 18.03 & $70-129$ & 103.00 & 10.33 & $78-119$ \\
\hline SCQ (out of 39) $)^{c}$ & 24.62 & 5.87 & $14-36$ & 3.12 & 2.72 & $1-10$ \\
\hline \multicolumn{7}{|l|}{ ADI-R scores ${ }^{c}$} \\
\hline Abnormal development $($ cutoff $=1)$ & 4.00 & 0.88 & $3-5$ & & & \\
\hline Social interaction $($ cutoff $=10)$ & 17.04 & 6.29 & $9-28$ & & & \\
\hline Communication (cutoff $=8$ ) & 13.83 & 4.34 & $8-22$ & & & \\
\hline Repetitive behaviors (cutoff $=3$ ) & 6.00 & 2.57 & $3-12$ & & & \\
\hline
\end{tabular}

Note-ASD, autism spectrum disorder. aRaven's Standard Progressive Matrices (Raven, Court, \& Raven, 1992). b beabody Picture Vocabulary Test-Third Edition (Dunn \& Dunn, 1997). 'Elevated scores reflect increased symptomatology. 


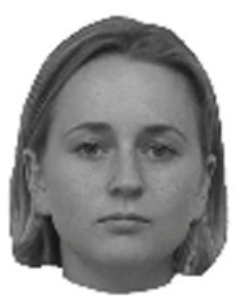

A

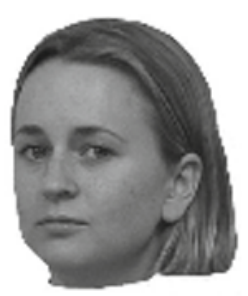

B

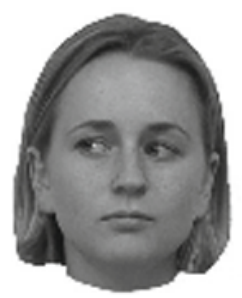

C

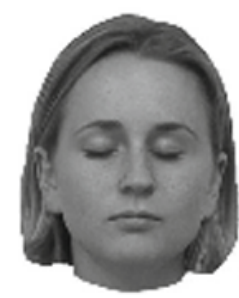

D

Figure 1. Example of stimuli presented in the direct-gaze, frontal-view condition (A), the direct-gaze, 3/4-view condition (B), the averted-gaze, frontal-view condition (C), and the eyes-closed condition (D).

Western Australia. All had been diagnosed independently by an experienced clinician using DSM-IV criteria (APA, 2000) and also either met full algorithm criteria on the Autism Diagnostic InterviewRevised (ADI-R; Lord, Rutter, \& Le Couteur, 1994; $n=19$ ) or scored 1 point below the threshold for autism $(n=5$; see Table 1 for scores). Three additional boys with ASD were tested ( $M=8$ years, 2 months) but were excluded from further analysis due to poor overall task performance $(<75 \%$ correct).

We recruited 24 typical children ( 20 boys; $M=10$ years, 0 months; $S D=1,8)$ from local mainstream schools. No typical child had a history of psychiatric or neurological disorder or displayed clinically significant levels of autistic symptomatology, as indexed by the Social Communication Questionnaire (SCQ; Rutter, Bailey, \& Lord, 2003), a screening tool for autism (see Table 1). There were no significant differences between the ASD and typical groups in chronological age $[F(1,47)=0.11, p=.74]$; nonverbal reasoning ability $[F(1,47)=0.51, p=.48]$, as assessed by Raven's Standard Progressive Matrices (Raven, Court, \& Raven, 1992); or receptivevocabulary ability $[F(1,47)=2.63, p=.11]$, as measured by the Peabody Picture Vocabulary Test, 3rd Edition (PPVT-III; Dunn \& Dunn, 1997; see Table 1). All 48 children obtained verbal and nonverbal IQ scores of at least 70 , reported normal or corrected-to-normal vision, and were free of medication.

The experiment had a 2 (group: ASD vs. typical) $\times 4$ (eye gaze: direct eye gaze, frontal view vs. direct eye gaze, $3 / 4$ view vs. averted eye gaze, frontal view vs. eyes closed, frontal view) mixed design with repeated measures on the second factor.

\section{Materials and Procedure}

The stimuli and procedure were similar to those in Macrae et al. (2002). Stimuli consisted of 48 black-and-white photographs of faces ( 24 female, 24 male) displaying neutral expressions. Of the 48 targets, 12 displayed direct gaze in frontal view, 12 displayed direct gaze in 3/4 view, 12 displayed averted gaze ( 6 left, 6 right) in frontal view, and 12 targets had their eyes closed (see Figure 1). Equal numbers of male and female targets were present in each condition.

Children were told that they would see a number of photographs of faces and were asked to identify, as quickly and accurately as possible, the sex of the face ("boy" or "girl") by making an appropriate keypress. Trials consisted of a central fixation cross $(2,400 \mathrm{msec})$, a blank screen $(30 \mathrm{msec})$, followed by the target face, which remained onscreen until children made a response or 1,200 msec had elapsed. Note that the eye region of each target face fell slightly above the central fixation cross, which did not, therefore, cue children's attention toward the eyes specifically. This procedural detail allowed us to examine the spontaneous effects of eye-gaze direction during children's gender judgments. All 48 trials were presented randomly at center screen on an Apple Macintosh G3 laptop computer. Accuracy and RTs were recorded.

Children were tested individually in a quiet room in their school and were seated approximately $50 \mathrm{~cm}$ from the computer screen. Their attention toward target stimuli was monitored carefully by the experimenter. Importantly, children were not informed as to the way in which the faces differed (i.e., eye gaze or head direction) and were never instructed explicitly to attend to the target's eye gaze. No feedback was given regarding performance. The session lasted approximately $40 \mathrm{~min}$, with the PPVT-III administered first, followed by the Raven's matrices and the sex-categorization task.

\section{RESULTS}

\section{Accuracy}

All children classified faces by sex with at least $75 \%$ accuracy. Accuracy data were submitted to a 2 (group) $\times$ 4 (eye gaze) mixed-model ANOVA. No significant effects emerged. Thus, gaze direction did not modulate the accuracy of person categorization, and children with ASD were just as accurate at categorizing faces by sex $(M=$ $87.8 \% ; S E=0.01)$ as were their typical counterparts $(M=89.3 \% ; S E=0.01) .^{1}$

\section{Response Latencies}

Mean categorization latencies on correct trials served as the dependent measure. ${ }^{2}$ Given the presence of outlying responses in the data set, RTs that were slower than $3 \mathrm{SDs}$ from the mean were excluded from the analysis $(4.5 \%$ of data). The data were submitted to a 2 (group) $\times 4$ (eye gaze) mixed model ANOVA. Although there was no main effect of group $(F<1)$, there was a significant effect of eye gaze $\left[F(3,138)=3.96, p<.01, \eta_{\mathrm{p}}^{2}=.08\right]$, which was qualified by a significant group $\times$ eye-gaze interaction $\left[F(3,138)=4.13, p<.01, \eta_{\mathrm{p}}^{2}=.08\right]$. (See Figure 2.) Subsequent within-group analyses revealed that sex-categorization performance in typical children was moderated by gaze direction $[F(3,69)=11.08, p<.001$, $\left.\eta_{\mathrm{p}}^{2}=.32\right]$, in such a way that RTs were faster when targets displayed direct gaze, as compared with averted gaze and eyes closed ( $t$ test, all $p s<.004)$. In contrast, gaze direction had no effect on task performance in the ASD group $[F(3,69)=0.03, p=.99]$. Children with ASD were as fast to categorize faces by sex when the eyes were directed straight ahead, averted, or closed.

There were no significant group differences in the speed with which children judged gender for any eye-gaze condition (all $p \mathrm{~s}>.15$ ). Also, individual differences in the effect of eye-gaze direction (mean $\mathrm{RT}_{\text {direct conditions }}-$ mean $\mathrm{RT}_{\text {averted/eyes-closed conditions }}$ ) were unrelated to autistic children's symptom scores on the ADI-R or SCQ. 


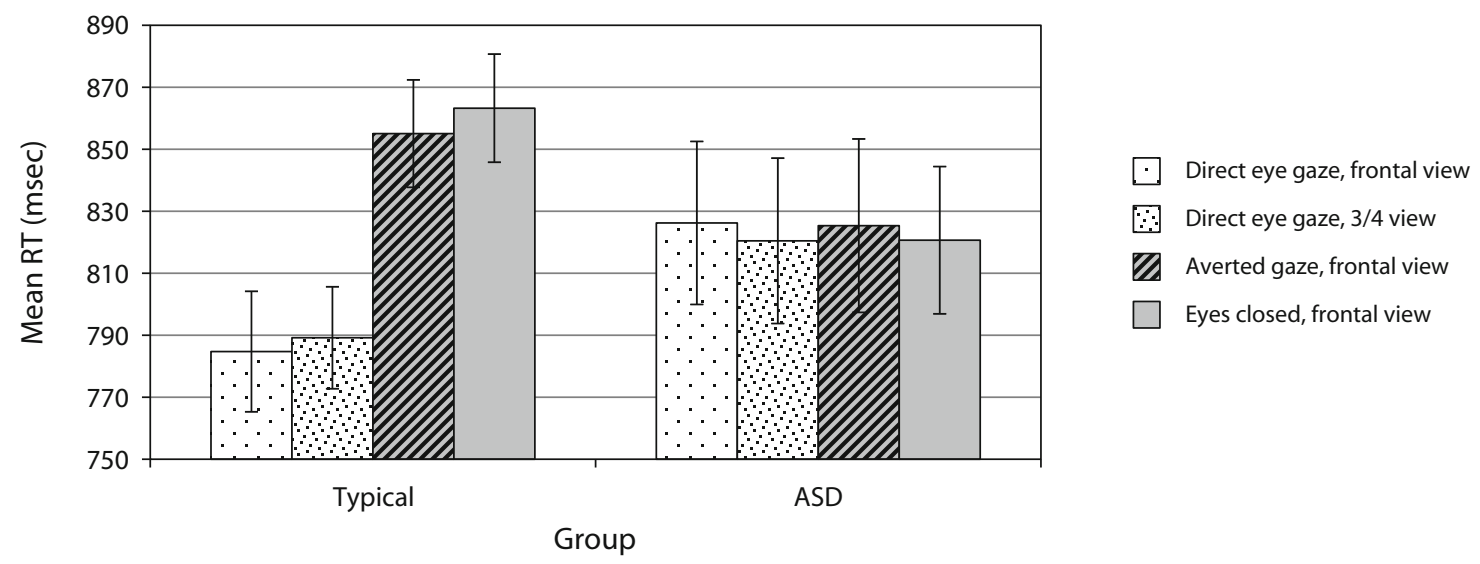

Figure 2. Mean response times (RTs, in milliseconds) for categorizing faces by sex as a function of group and gaze direction. Error bars represent \pm 1 standard error of the mean. ASD, autism spectrum disorder.

\section{DISCUSSION}

In this study, we sought to determine whether gaze direction modulates a basic person-perception process (i.e., sex categorization) for typical children and children with ASD in the way it does for typical adults (Macrae et al., 2002). The results were revealing. Although direct eye gaze enhanced the speed of categorical judgments in typical school-age children, it did not facilitate the speed with which children with ASD classified faces by sex. In fact, the sex-categorization times of children with ASD were similar across all conditions, indicating that children were unaffected by the gaze direction of the targets.

The lack of an advantage for direct gaze in the ASD group cannot be attributed to difficulties perceiving gender, since children with ASD were just as accurate at categorizing faces as were age- and ability-matched comparison children. Nor can these findings be a result of the overall slower RTs usually characteristic of this population (e.g., Behrmann et al., 2006), inasmuch as the RTs across all four eye-gaze conditions for the ASD group were well within the range of RTs of typical children. Moreover, both aspects of the results attest to children's attention and motivation to the task. Also, it is unlikely that results are due to fundamental difficulties discriminating eye-gaze direction, since it is well established that children with autism can accurately make such judgments when deviations in gaze are of the size used in the present study (e.g., Baron-Cohen et al., 1995; Wallace et al., 2006).

Here, children were asked to judge, as quickly as possible, the gender of faces - a key component of the personperception process. Because eye-gaze impairments previously have been argued to be rooted in the inability to understand fully the intentional nature of gaze cues, and thus related to their sociocommunicative functions (e.g., Baron-Cohen, 1995), we anticipated that gaze direction might affect the efficiency with which children with ASD extract basic person-relevant information from faces. The fact that it did not suggests that gaze processing impairments in autism may extend beyond a primary problem in interpreting eye gaze in sociocommunicative (i.e., men- talistic) contexts. The present findings suggest that children with autism miss out both on crucial aspects of social communication (e.g., joint shared attention) and on the other important advantages that mutual gaze affords.

What might drive children's failure to benefit from direct eye gaze during the person-construal process? One possibility is that they do not fixate the eye region of the face. Several reports have demonstrated that individuals with ASD fixate the eyes less than typical individuals do during viewing of facial images (e.g., Pelphrey et al., 2002), but this finding is not uncontroversial. Other studies have reported typical spontaneous attention toward the eyes in autism (e.g., Fletcher-Watson et al., 2009; see Senju \& Johnson, 2009, for a discussion), and one study has shown that children with autism make as many fixations to eyes as do typical children during a gender identification task (Best, Dundas, \& Strauss, 2009). Although our observation of autistic children's gaze during testing confirmed good inspection, in future studies, children's eye movements might be monitored during task performance to rule out this possibility.

A second possibility is that children with ASD attend less to those faces showing direct eye gaze specifically. Some researchers (e.g., Dalton et al., 2005) have proposed that individuals with autism actively avoid faces "looking at me" because direct eye gaze elicits negative arousal. Reduced attention toward mutual gaze in particular should, in turn, have deleterious effects for subsequent information processing. On this view, then, one should expect that autistic children's gender judgments might be slower for faces showing direct, as compared with averted, gaze or eyes closed. Yet our sample of children with ASD showed comparable RTs for gender judgments in both direct and averted gaze conditions. Our findings parallel those showing similar processing of direct and averted eye gaze in behavioral (Wallace et al., 2006), eyetracking (Hernandez et al., 2009), and event-related brain potential (Senju, Tojo, Yaguchi, \& Hasegawa, 2005) studies, which together speak against the possibility that the present findings are due to hyperarousal to direct eye gaze in autism. 
We suggest instead that eye gaze does not modulate subsequent person-construal processes for children with ASD. Clearly, in many different ways, someone looking at you can be critically different from someone looking away (Mason, Tatkow, \& Macrae, 2005). Establishing the meaning conveyed by direct eye gaze (e.g., attraction, aggression, friendliness) leads to more elaborate encoding of a person's face (identity, expression, sex). Functional imaging studies provide converging evidence that faces with direct eye gaze warrant deeper encoding: Faces displaying direct eye gaze elicit greater neural activity in "social-brain" areas than do those displaying averted eye gaze for numerous different person-perception judgments (see Nummenmaa \& Calder, 2009, for a review). Indeed, George, Driver, and Dolan (2001) reported that, during performance of a gender discrimination task, eye-gaze direction (regardless of head orientation) modulated the connectivity or temporal coupling of activity between the amygdala and fusiform cortex. Their findings fit with a new proposal suggesting that subcortical structures (including the amygdala), together with contextual information modulated by prefrontal cortical regions, modulate activity in key cortical structures of the social-brain network (Senju \& Johnson, 2009). It is possible, therefore, that the failure to find evidence of an eye-contact effect in our sample of children with ASD might be attributable to reduced connectivity between "fast-track" subcortical structures and social-brain regions in response to perceived eye contact.

Findings from recent reflexive orienting studies further support the notion that the difficulties in autism might reflect a failure to understand the "significance of the eyes" in this broader sense. Unlike typical individuals whose attentional orienting is more strongly reflexive in response to gaze than to nongaze cues (Friesen, Ristic, \& Kingstone, 2004), individuals with autism do not show this preferential sensitivity to gaze (e.g., Senju, Tojo, Dairoku, \& Hasegawa, 2004). Ristic et al. (2005) argued that individuals with autism may rely on physical stimulus characteristics (e.g., apparent motion and/or low-level visual analysis) when learning cue-target contingencies, rather than direction of eye gaze per se, reflecting a lack of appreciation of eye-gaze direction as an inherently social cue. The present findings provide corroborating evidence for this view: Although typical children's gender judgments were affected by irrelevant gaze direction, the responses of autistic children were not, suggesting that mutual eye gaze does not hold special significance for those with autism.

What is clear from our study is that autistic individuals' difficulties with eye gaze go beyond the disadvantages described in previous studies, to encompass more basic features, such as person perception. Further research should aim to elucidate the precise neurological mechanisms that underlie these broader social disadvantages.

\section{AUTHOR NOTE}

During this research, C.N.M. was supported by a Royal Society Wolfson Award. We thank the children, teachers, and families for their support, Anneli Kylliäinen for reading an earlier draft of the manuscript, and
Marc Stears and Gill Rhodes for lively theoretical discussions. Address correspondence to E. Pellicano, Centre for Research in Autism and Education, Department of Psychology and Human Development, Institute of Education, 25 Woburn Square, London WC1H 0AA, England (e-mail: 1.pellicano@ioe.ac.uk).

\section{REFERENCES}

American Psychiatric Association (2000). Diagnostic and statistical manual of mental disorders (4th ed., text rev.). Washington, DC: Author.

Bakti, A., Baron-Cohen, S., Wheelwright, S., Connellan, J., \& Ahluwalia, J. (2000). Is there an innate gaze module? Evidence from human neonates. Infant Behavior \& Development, 23, 223-229. doi:10.1016/S0163-6383(01)00037-6

Baron-Cohen, S. (1995). Mindblindness: An essay on autism and theory of mind. Cambridge, MA: MIT Press.

Baron-Cohen, S., Campbell, R., Karmiloff-Smith, A., Grant, J., \& WALKer, J. (1995). Are children with autism blind to the mentalistic significance of the eyes? British Journal of Developmental Psychology, 13, 379-398.

Baron-Cohen, S., Cox, A., Baird, G., Swettenham, J., NightinGale, N., Morgan, K., ET AL. (1996). Psychological markers in the detection of autism in infancy in a large population. British Journal of Psychiatry, 168, 158-163.

Baron-Cohen, S., Jolliffe, T., Mortimore, C., \& Robertson, M. (1997). Another advanced test of theory of mind: Evidence from very high functioning adults with autism or Asperger syndrome. Journal of Child Psychology \& Psychiatry, 38, 813-822. doi:10.1111/ j.1469-7610.1997.tb01599.x

Behrmann, M., Avidan, G., Leonard, G. L., Kimchi, R., Luna, B., Humphreys, K., \& Minshew, N. (2006). Configural processing in autism and its relationship to face processing. Neuropsychologia, $\mathbf{4 4}$, 110-129. doi:10.1016/j.neuropsychologia.2005.04.002

Best, C. A., Dundas, E. M., \& Strauss, M. S. (2009, April). Identifying gender information in morphed faces: An eye tracking study of individuals with autism. Poster presented at the Biennial Meeting of the Society for Research in Child Development, Denver.

Dalton, K. M., Nacewicz, B. M., Johnstone, T., Schaefer, H. S., Gemsbacher, M. A., Goldsmith, H. H., et AL. (2005). Gaze-fixation and the neural circuitry of face processing in autism. Nature Neuroscience, 8, 519-526. doi:10.1038/nn1421

Dunn, L. M., \& Dunn, L. M. (1997). PPVT-III: Peabody Picture Vocabulary Test (3rd ed.). Circle Pines, MN: American Guidance Service.

Farroni, T., Csibra, G., Simion, G., \& Johnson, M. H. (2002). Eye contact detection in humans from birth. Proceedings of the National Academy of Sciences, 99, 9602-9605. doi:10.1073/pnas.152159999

Farroni, T., Mansfield, E. M., LaI, C., \& Johnson, M. H. (2003). Infants perceiving and acting on the eyes: Tests of an evolutionary hypothesis. Journal of Experimental Child Psychology, 85, 199-212. doi:10.1016/S0022-0965(03)00022-5

Farroni, T., Massaccesi, S., Menon, E., \& Johnson, M. H. (2007). Direct gaze modulates face recognition in young infants. Cognition, 102, 396-404. doi:10.1016/j.cognition.2006.01.007

Fletcher-Watson, S., Leekam, S. R., Benson, V., Frank, M. C., \& FINDLAY, J. M. (2009). Eye-movements reveal attention to social information in autism spectrum disorder. Neuropsychologia, 47, 248257. doi:10.1016/j.neuropsychologia.2008.07.016

Friesen, C. K., Ristic, J., \& Kingstone, A. (2004). Attentional effects of counterpredictive gaze and arrow cues. Journal of Experimental Psychology: Human Perception \& Performance, 30, 319-329.

George, N., Driver, J., \& Dolan, R. J. (2001). Seen gaze-direction modulates fusiform activity and its coupling with other brain areas during face processing. NeuroImage, 13, 1102-1112. doi:10.1006/ nimg.2001.0769

Hains, S. M. J., \& Muir, D. W. (1996). Infant sensitivity to adult eye direction. Child Development, 67, 1940-1951. doi:10.1111/j.1467-8624 1996.tb01836.x

Hernandez, N., Metzger, A., Magnè, R., Bonnet-Brilhault, F., Roux, S., Barthelemy, C., \& Martineau, J. (2009). Exploration of core features of a human face by healthy and autistic adults analyzed by visual scanning. Neuropsychologia, 47, 1004-1012. doi:10.1016/ j.neuropsychologia.2008.10.023 
Hood, B. M., Macrae, C. M., Cole-Davies, V., \& Dias, M. (2003). Eye remember you: The effects of gaze direction on face recognition in children and adults. Developmental Science, 6, 67-71. doi:10.1111/1467-7687.00256

Leekam, S. R., Baron-Cohen, S., Perrett, D., Milders, M., \& Brown, S. (1997). Eye-direction detection: A dissociation between geometric and joint attention skills in autism. British Journal of Developmental Psychology, 15, 77-95.

Leekam, S. R., Hunnisett, E., \& Moore, C. (1998). Targets and cues: Gaze-following in children with autism. Journal of Child Psychology \& Psychiatry, 39, 951-962. doi:10.1111/1469-7610.00398

Lord, C., Rutter, M., \& Le Couteur, A. (1994). Autism Diagnostic Interview-Revised: A revised version of a diagnostic interview for caregivers of individuals with possible pervasive developmental disorders. Journal of Autism \& Developmental Disorders, 24, 659-685. doi:10.1007/BF02172145

Macrae, C. N., \& Bodenhausen, G.V. (2000). Social cognition: Thinking categorically about others. Annual Review of Psychology, 51, 93120. doi:10.1146/annurev.psych.51.1.93

Macrae, C. N., Hood, B. M., Milne, A. B., Rowe, A. C., \& Mason, M. F. (2002). Are you looking at me? Eye gaze and person perception. Psychological Science, 13, 460-464. doi:10.1111/1467-9280.00481

Mason, M. F., Hood, B. M., \& Macrae, C. N. (2004). Look into my eyes: Gaze direction and person memory. Memory, 12, 637-643. doi:10.1080/09658210344000152

Mason, M. F., Tatkow, E. P., \& Macrae, C. N. (2005). The look of love: Gaze shifts and person perception. Psychological Science, 16, 236-239. doi:10.1111/j.0956-7976.2005.00809.x

Nation, K., \& Penny, S. (2008). Sensitivity to eye gaze in autism: Is it normal? Is it automatic? Is it social? Development \& Psychopathology, 20, 79-97. doi:10.1017/S0954579408000047

NummenmaA, L., \& CALDER, A. J. (2009). Neural mechanisms of social attention. Trends in Cognitive Sciences, 13, 135-143. doi:10.1016/ j.tics.2008.12.006

Pelphrey, K. A., Sasson, N. J., Reznick, J. S., Paul, G., GoldMAN, B. D., \& Piven, J. (2002). Visual scanning of faces in autism. Journal of Autism \& Developmental Disorders, 32, 249-261. doi:10.1023/A:1016374617369

Raven, J. C., Court, J. H., \& Raven, J. (1992). Standard progressive matrices. Oxford: Oxford Psychologists Press.

Ristic, J., Mottron, L., Friesen, C. K., Iarocci, G., Burack, J. A., \& Kingstone, A. (2005). Eyes are special but not for everyone: The case of autism. Cognitive Brain Research, 24, 715-718. doi:10.1016/ j.cogbrainres.2005.02.007
Rutter, M., Bailey, A., \& Lord, C. (2003). SCQ: Social Communication Questionnaire. Los Angeles: Western Psychological Services.

Senju, A., \& Csibra, G. (2008). Gaze following in human infants depends on communicative signals. Current Biology, 18, 668-671. doi:10.1016/j.cub.2008.03.059

Senju, A., Csibra, G., \& Johnson, M. H. (2008). Understanding the referential nature of looking: Infants' preference for object-directed gaze. Cognition, 108, 303-319. doi:10.1016/j.cognition.2008.02.009

Senju, A., \& Johnson, M. H. (2009). Atypical eye contact in autism: Models, mechanisms and development. Neuroscience \& Biobehavioral Reviews, 33, 1204-1214. doi:10.1016/j.neubiorev.2009.06.001

Senju, A., Tojo, Y., Dairoku, H., \& Hasegawa, T. (2004). Reflexive orienting in response to eye gaze and an arrow in children with and without autism. Journal of Child Psychology \& Psychiatry, 45, 445458. doi:10.1111/j.1469-7610.2004.00236.X

Senju, A., Tojo, Y., Yaguchi, K., \& Hasegawa, T. (2005). Deviant gaze processing in children with autism: An ERP study. Neuropsychologia, 43, 1297-1306. doi:10.1016/j.neuropsychologia.2004.12.002

Sigman, M., Mundy, P., Sherman, T., \& Ungerer, J. (1986). Social interactions of autistic, mentally retarded and normal children and their caregivers. Journal of Child Psychology \& Psychiatry, 27, 647655. doi:10.1111/j.1469-7610.1986.tb00189.x

TAN, J., \& HARRIS, P. (1991). Autistic children understand seeing and wanting. Development \& Psychopathology, 3, 163-174. doi:10.1017/ S0954579400000055

VON Grünau, M., \& Anston, C. (1995). The detection of gaze direction: A stare-in-the-crowd effect. Perception, 24, 1297-1313. doi:10.1068/ p241297

Wallace, S., Coleman, M., Pascalis, O., \& Bailey, A. (2006). A study of impaired judgment of eye-gaze direction and related faceprocessing deficits in autism spectrum disorders. Perception, 35, 1651-1664. doi:10.1068/p5442

\section{NOTES}

1. This analysis was rerun to include the 3 children with ASD who obtained very poor sex classification scores: No significant group differences in accuracy emerged $[F(1,49)=1.42, p=.24]$.

2. Analyses using median RT as the dependent measure revealed comparable results.

(Manuscript received May 5, 2009;

revision accepted for publication August 17, 2009.) 